\title{
Mucormycosis an Emerging Fungal Infection Associated with Global COVID-19 Pandemic
}

\section{Singh $\mathrm{P}^{1 *}$, Malik $\mathrm{S}^{2}$ and Lal $\mathrm{V}^{3}$}

${ }^{1}$ Senior research Scientist, Department of Microbiology and Serology, Delhi, India

${ }^{2}$ Technical director, Department of Microbiology and Serology, Dr Lal Path Labs, National

Reference Laboratory, Delhi, India

${ }^{3}$ Executive director, Dr Lal Path Labs National Reference Laboratory, Delhi, India

*Corresponding author: Puneeta Singh, Sr. Scientist, Department of Microbiology and Serology, Dr Lal Path Labs, National Reference Laboratory, Rohini, Delhi, India, Email: puneeta.singh@lalpathlabs.com

\section{Short Communication}

Mucormycosis, also known as black fungus caused by fungi in the order Mucorales. In most cases, it is due to an invasion of the genera Rhizopus and Mucor, common bread molds. Most common infections caused by Rhizopus oryzae. It is less likely due to Lichtheimia, and rarely due to Apophysomyces. Others include Cunninghamella, Mortierella, and Saksenaea.

Mucormycosis is usually rare but rise in cases particularly noted in India in COVID-19 Pandemic, estimated to be about 70 times higher than in the rest of the world [1-4]. People of young age may affect, including young adults. This association thought to relate to reduced immune function during the course of the illness and related to glucocorticoid therapy for COVID-19.

Mucormycosis has reported in place intravenous catheters, Outbreaks have also linked to hospital bed sheets, negative-pressure rooms, water leaks, poor ventilation, contaminated medical equipment, and building works. It most commonly infects the nose, sinuses, eye and brain resulting in a runny nose, one sided facial swelling and pain, headache, fever, blurred vision, swollen and bulging eye, and tissue death [4,5]. Other forms of disease may infect the lungs, stomach and intestines, and skin. It cause disease only in some people with high risk factors include diabetes with persistently high blood sugar levels or diabetic ketoacidosis, kidney problems, long-term steroids or immunosuppressant use, and to a lesser extent in HIV/AIDS [5].

Diagnosis requires identifying the mold in the affected tissue by biopsy and confirming it with a fungal culture [4]. Tests may also include culture and direct detection of the fungus in lung fluid, blood, Sputum, nasal discharge, and urine. Treatment is generally with amphotericin B and surgical debridement [5].

This study aimed at determining the Mucor species associated with COVID-19 Pandemic in diverse clinical specimens tested between second waves of COVID-19 in Delhi during April 2021 to July 2021 performed at Microbiology department of Dr Lal Path Labs. Mold species were cultured by Conventional (culture) and identified by MALDI-TOF.

Out of 59 isolates of Mucormycosis most commonly isolates were Rhizopus oryzae constituted (37, 62.7\%), followed by Rhizopus microsporus $(19,32.2 \%)$, Lichtheimia (Mucor spp.) $(3,5.1 \%)$ over a period of four month. 59 isolates of Mucormycosis was most commonly isolated in Nasal cavity constituted (40.7\%) and least Body fluids (5.1\%) over a period of 4 month constituted as per (Figure 1). low white cells, cancer, organ transplant, iron overload, 



Figure 1: Prevalence of Mucormycosis in different clinical samples.

Out of the 59 isolates, $20.3 \%$ of isolates of them belonging to the age group of 31-40 years and Rest were $79.7 \%$ of $41-99$ years. In our study the most predominant age group infected with Mucormycosis were elderly adults $>=61$ (42.4\%) (Figure 2).

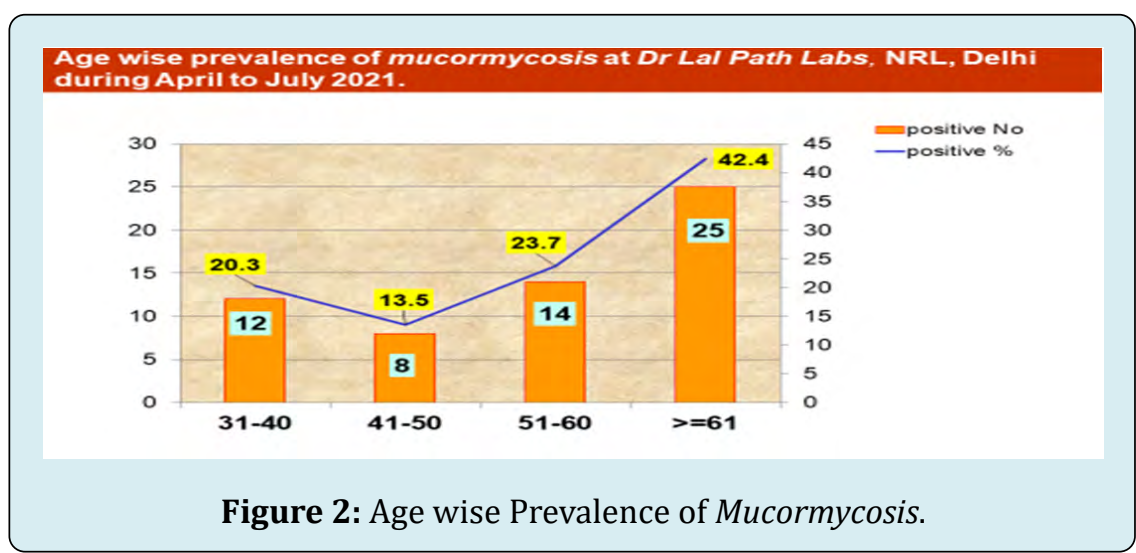

\section{Conclusion}

The fungal pathogens has changed over a period with new species emerging as well as old species increasing become more virulent and resistant to primary antifungal drugs. We should be concern about the national emergence and the challenges in managing the rare fungal infection cases like Mucormycosis that obtained in diverse clinical situations from patients with COVID-19 during second wave of pandemic. Additional studies are required to assess the optimal duration of therapy as well as the specific roles of amphotericin B and the newer triazoles posaconazole in the treatment of mucormycosis.

\section{References}

1. Prakash H, Chakrabarti A (2021) Epidemiology of
Mucormycosis in India. Microorganisms 9(3): 523.

2. Raut A, Huy NT (2021) Rising incidence of mucormycosis in patients with COVID-19: another challenge for India admidst the second wave?. Respiratory Medicine 9(8): E77.

3. Skiada A, Pavleas I, Drogari-Apiranthitou M (2020) Epidemiology and Diagnosis of Mucormycosis: An Update. J Fungi 6(4): 265.

4. Stone N, Gupta N, Schwartz I (2021) Mucormycosis: time to address this deadly fungal infection. The Lancet Microbe 2(8): E343-E344.

5. Reid G, Lynch JP, Fishbein MC, Clark NM (2020) Mucormycosis. Semin Respir Crit Care Med 41(1): 99114.

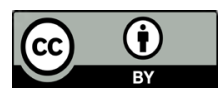

